\title{
IMAGE SUPER RESOLUTION USING SPARSE NEIGHBOR EMBEDDING AND CLUSTERING ALGORITHM
}

\author{
Dr. Dharmanna $\mathrm{L}^{1}$ and Deekshith $\mathrm{K}^{2}$
}

Abstract : This paper presents a new approach to image super resolution, based upon Sparse Neighbor Embedding and clustering algorithm .Consider a low resolution image as input. Then do LR patch separation, followed by feature extraction using histograms of oriented gradients (HOG). Then perform classification using K-means clustering algorithm. Then perform Sparse Neighbor Embedding. Finally synthesize HR patches and subject it to de-blurring algorithm to obtain a Super-Resolution Image.

Key words: Super Resolution, Sparse neighbor embedding, histograms of oriented gradients, KMeans clusters, K-NN Classification

\section{INTRODUCTION}

The central aim of Super-Resolution (SR) is to generate a higher resolution image from lower resolution images. High resolution image offers a high pixel density and thereby more details about the original scene. The need for high resolution is common in computer vision applications for better performance in pattern recognition and analysis of images. High resolution is of importance in medical imaging for diagnosis. Many applications require zooming of a specific area of interest in the image wherein high resolution becomes essential, e.g. surveillance, forensic and satellite imaging applications.

However, high resolution images are not always available. This is since the setup for high resolution imaging proves expensive and also it may not always be feasible due to the inherent limitations of the sensor, optics manufacturing technology. These problems can be overcome through the use of image processing algorithms, which are relatively inexpensive, giving rise to concept of super-resolution. It provides an advantage as it may cost less and the existing low resolution imaging systems can still be utilized.

Image super-resolution (SR) reconstruction is the process of generating an image at a higher spatial resolution by using one or more low-resolution (LR) inputs from a scene.

Over more than two decades, many methods for SR reconstruction have been proposed, which can broadly be grouped into three categories: interpolation-based methods, multi image based methods, and example-learning-based methods. In this project, we focus on the NE-based methods. NE-based SR algorithms can represent more patterns even if a relatively smaller training data set is available and thus

${ }^{1}$ Dept. Of Ise, Sdmit

${ }^{2}$ Dept. Of Ise, Sdmit 
show much stronger generalization ability for a variety of images. The reconstruction process takes place in the following stages:

1) Both the HOG and the first and second-order gradient features are extracted in a raster-scan order from the up scaled version of the LR input by using the bicubic (BI) interpolation with a factor of 2 .

2) Subset selection that the HOG feature of each LR input matches the centroids of clusters is performed to find a medium-scale subset close to the LR input for synthesis process.

3) SpNE is applied to synthesize the HR image patch of the LR input, in which searching neighbors and estimating weights are simultaneously conducted.

4) After constructing all the HR patches and obtaining the initial HR image, the total-variation-based (TV) deblurring and the iterative back-projection (IBP) algorithm are sequentially performed to obtain the final HR outcome.

\section{DETAILED METHODOLOGY}

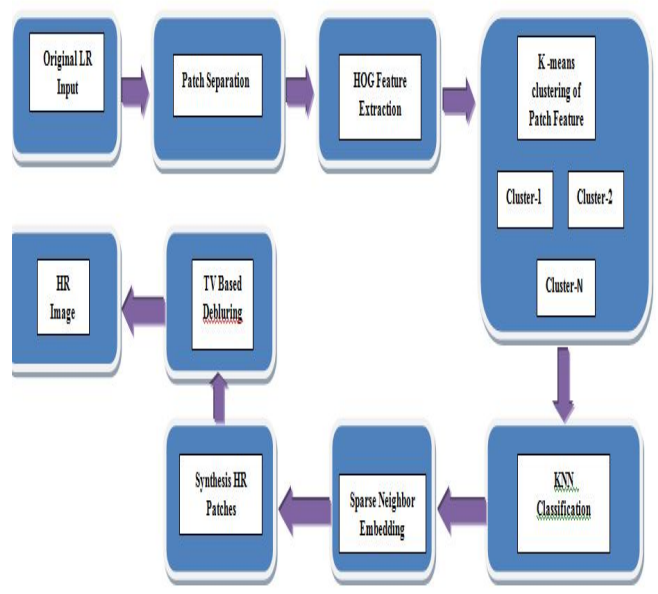

2.1 Modules

Figure 2.1: Block diagram showing the system architecture

- Patch Separation

- Feature Extraction

- K-Means Clusters

- K-NN Classification

- Sparse neighbor embedding

- Synthesize HR patches

- TV Deblurring

\subsection{Description}

Patch Separation:

The whole image matrix is decomposed into patch matrix. These patches forms a sub matrix namely $(15 * 15)$ pixels. 


\section{Feature Extraction:}

Local object appearance and shape can often be characterized rather well by the distribution of local intensity gradients or edge direction. HOG features are calculated by taking orientation histograms of edge intensity in local region. Specially, we can divide an image into N local regions called "Patch". Extract HOG features form each patch. We choose HOG rather than other low-level features, such as pixel intensities, gradient information or a combination of both. By contrast, the HOG feature does not have either problem.

\section{K-Means Clusters:}

The Patch features are subjected to K-Means Clustering. It clusters each patch features depending on the centroids. Finally, it provides a label for each clustered patch, which can be used to train the classifier. Geometric Clustering:

Unsupervised learning refers to the problem of trying to find hidden structure in unlabeled data. Clustering is a technique for finding similarity groups in data, called clusters. I.e.,it groups data instances that are similar to (near) each other in one cluster and data instances that are very different (far away) from each other into different clusters. Clustering is often called an unsupervised learning task as no class values denoting an a priori grouping of the data instances are given, which is the case in supervised learning.

Due to historical reasons, clustering is often considered synonymous with unsupervised learning.

- K-means is a partitional clustering algorithm

- Let the set of data points (or instances) D be

$$
\left\{\mathrm{x}_{1}, \mathrm{x}_{2}, \ldots, \mathrm{x}_{\mathrm{n}}\right\}
$$

where $\mathrm{x}_{\mathrm{i}}=\left(\mathrm{x}_{\mathrm{i} 1}, \mathrm{x}_{\mathrm{i} 2}, \ldots, \mathrm{x}_{\mathrm{ir}}\right)$ is a vector in a real-valued space $\mathrm{X} \subseteq \mathrm{R}^{\mathrm{r}}$, and $\mathrm{r}$ is the number of attributes (dimensions) in the data.

* The k-means algorithm partitions the given data into k clusters.

- Each cluster has a cluster center, called centroid.

- $\mathrm{k}$ is specified by the user

* Given k, the k-means algorithm works as follows:

1) Randomly choose $\mathrm{k}$ data points (seeds) to be the initial centroids, cluster centers

2) Assign each data point to the closest centroid

3) Re-compute the centroids using the current cluster memberships.

4) If a convergence criterion is not met, go to 2).

K-NN Classifier:

The cluster labels are used to train the classifier. It contains ID for the patch. Dimension reduced features of patch in order to use less storage space. K-NN using the Euclidean Distance predicts the nearest neighbor for the test patch. Place items in class to which they are "closest". Must determine distance between an item and a class. 
- Classes represented by

- Centroid: Central value.

- Medoid: Representative point.

- Individual points

Sparse neighbor embedding:

This provides a sparse representation of the over complete dictionary. By modifying the Robust-SL0 algorithm.Robust-SL0, enjoying the same computational advantages of SL0, while demonstrating remarkable robustness against noise. The algorithm is developed by adopting the corresponding optimization problem for noisy settings, followed by theoretically-justified approximation to reduce the complexity.

Image SR with Sparse NE

Input:

- The LR image $\mathrm{I}_{1}$ and the size of LR patch sxs ;

- The neighborhood size and an integer;

- The HOG centroid set G obtained by K-means clustering;

- The training data set $\mathrm{X}_{\mathrm{S}}$ and $\mathrm{Y}_{\mathrm{S}}$

Output:

- HR image $\mathrm{X}^{*}$

Synthesize HR patches:

On solving the optimization problem, synthesize the HR patches.

TV Deblurring:

Perform the TV-based deblurring on the initial estimation and obtain the deblurred HR image. The TVbased method is capable of suppressing the ringing effects.

\section{EXPERIMENTAL RESULTS}

In this paper we have enhanced a low resolution image to high resolution image. This method mainly contains 5 steps this are shown from figure 1 to figure 6.

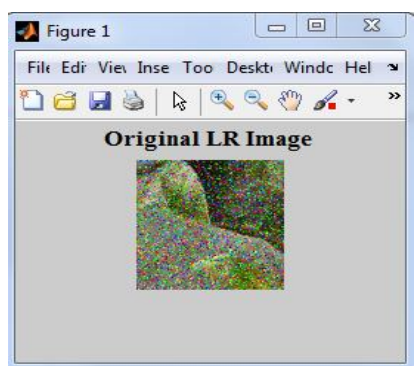

Figure 1: Original Low Resolution Image 


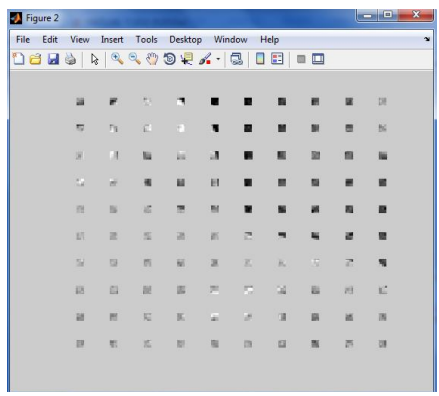

Figure 2: Patch Separation of Low Resolution Image

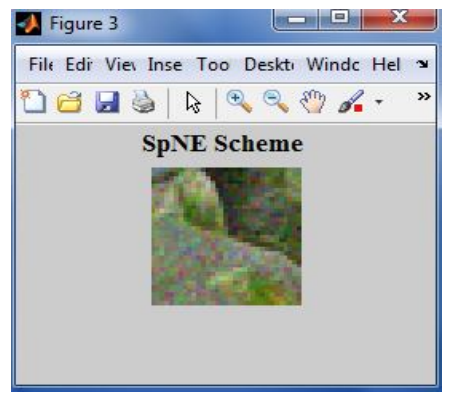

Figure 3:Sparse Neighbor Embedding Scheme

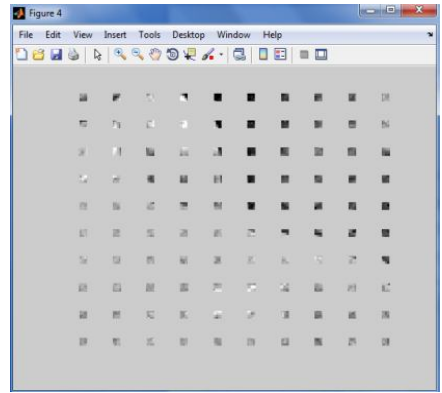

Figure 4: Patch Separation of High Resolution Image

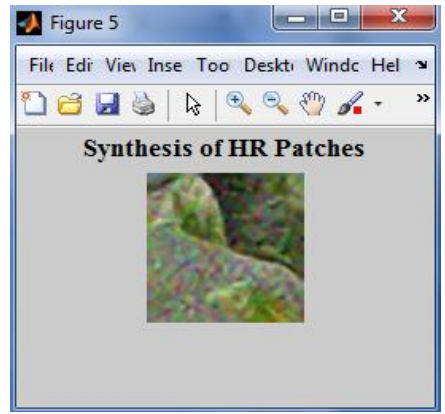

Figure 5:Synthesis of High Resolution Patches 


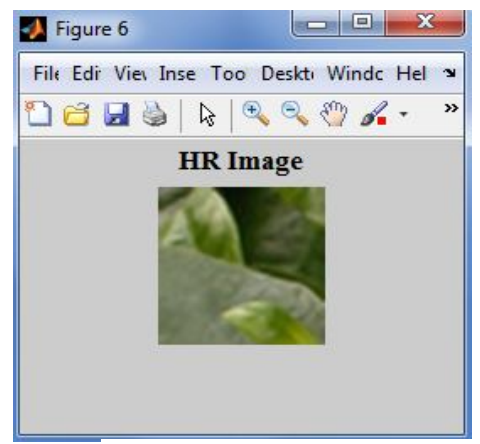

Figure 6:High Resolution Image

\section{CONCLUSION}

The aim of the project is to obtain high resolution image from low resolution image.

The proposed method is expected to achieve competitive super resolution quality compared with other state of the art baselines.

\section{REFERENCES}

[1]. Xinbo Gao,Kaibing Zhang,Dacheng Tao "Image Super-Resolution With Sparse Neighbor Embedding" IEEE Transactions on Image Processing Issue $7 \bullet$ July 2012

[2]. S. Park, M. Park, and M. Kang, "Super-resolution image reconstruction: A technical overview," IEEE Signal Process. Mag., vol. 20, no.3, pp. 21-36, May 2003.

[3]. X. Li and M. T. Orchard, "New edge-directed interpolation," IEEE Trans. Image Process., vol. 10, no. 10, pp. 1521-1527, Oct. 2001.

[4]. N. A. Dodgson, "Quadratic interpolation for image resampling," IEEE Trans. Image Process., vol. 6, no. 9, pp. 1322-1326, Sep. 1997.

[5]. L. Zhang and $\mathrm{X}$. Wu, "An edge-guided image interpolation algorithm via directional filtering and data fusion,” IEEE Trans. Image Process.,vol. 15, no. 8, pp. 2226-2238, Aug. 2006.

[6]. W. T. Freeman, T. R. Jones, and E. C. Pasztor, "Example-based superresolution, "IEEE Comput. Graph. Appl., vol. 22, no. 2, pp. 56-65, Mar./Apr. 2002.

[7]. H. Chang, D.-Y. Yeung, and Y. Xiong, "Super-resolution through neighbor embedding," in Proc. IEEE Conf. Comput. Vis. Pattern Recog., Jul. 2004, pp. 275-282.

[8]. X. Li, Y. Hu, X. Gao, D. Tao, and B. Ning, "A multi-frame image super-resolution method," Signal Process., vol. 90, no. 2, pp. 405-414,Feb. 2010.

[9]. M. Protter,M. Elad, H. Takeda, and P. Milanfar, "Generalizing the nonlocal-means to super-resolution reconstruction,” IEEE Trans. ImageProcess., vol. 18, no. 1, pp. 36-51, Jan. 2009.

[10]. X. Gao, Q. Wang, X. Li, D. Tao, and K. Zhang, "Zernike-moment based image super resolution," IEEE Trans. Image Process., vol. 20,no. 10, pp. 2738-2747, Oct. 2011.

[11]. R. Chan, S. Riemensch neider, L. Shen, and Z. Shen, "Tight Frame: An Efficient Way for High-Resolution Image Reconstruction," Applied and Computational Harmonic Analysis, vol. 17, pp. 91-115, 2004

[12]. M. Eland and A. Feuer, "Restoration of a Signal Super-Resolution Image from Several Blurred, Noisy and Undersampled Measured Images,” IEEE Trans. Image Processing, pp. 1646-1658, 1997. 